\title{
Analysis of functionally graded rotating disks with variable thickness
}

\begin{abstract}
Elastic solutions for axisymmetric rotating disks made of functionally graded material with variable thickness are presented. The material properties and disk thickness profile are assumed to be represented by two power-law distributions. In the case of hollow disk, based on the form of the power-law distribution for the mechanical properties of the constituent components and the thickness profile function, both analytical and semi-analytical solutions are given under free-free and fixed-free boundary conditions. For the solid disk, only semianalytical solution is presented. The effects of the material grading index and the geometry of the disk on the stresses and displacements are investigated. It is found that a functionally graded rotating disk with parabolic or hyperbolic convergent thickness profile has smaller stresses and displacements compared with that of uniform thickness. It is seen that the maximum radial stress for the solid functionally graded disk with parabolic thickness profile is not at the centre like uniform thickness disk. Results of this paper suggest that a rotating functionally graded disk with parabolic concave or hyperbolic convergent thickness profile can be more efficient than the one with uniform thickness.
\end{abstract}

Keyword: Rotating disk, Variable thickness, Elasticity, Functionally graded material 